\title{
Development of size constancy in children: A test of the proximal mode sensitivity hypothesis
}

\author{
CARL E. GRANRUD and TRISTI T. N. SCHMECHEL \\ University of Northern Colorado, Greeley, Colorado
}

\begin{abstract}
In two experiments, we attempted to replicate Shallo and Rock's (1988) finding that 5- and 6-yearold children exhibit size constancy for a distant object when tested with comparison objects that are matched for visual angle. Experiment $1(N=80)$ included four age groups: 5-, 6-, and 9-year-olds and adults. Participants viewed one standard object from $61 \mathrm{~m}$ and indicated which of nine nearby comparison objects matched the standard object in size. The comparison objects subtended equal visual angles in one condition and different visual angles in another. In both conditions, the 5- and 6-year-old children underestimated the size of the standard object, whereas the 9-year-old children and adults made nearly accurate size estimates. In Experiment $2(N=32)$, we replicated the finding that 6 -yearold children underestimate size when tested with comparison objects that subtend equal visual angles. Our results conflict with those of Shallo and Rock and support earlier findings that young children do not exhibit size constancy for distant objects.
\end{abstract}

A consistent pattern has emerged from research on size constancy in children. In several studies, it has been reported that children as young as $4-5$ years of age can accurately estimate an object's size when the object observed is within a few meters but that young children's size estimates are less accurate than those of adults when objects are more distant. For example, at distances of 30.5 to $61 \mathrm{~m}$, adults typically make nearly accurate size estimates, whereas children under 9 years of age significantly underestimate objects' sizes (Brislin \& Leibowitz, 1970; Leibowitz, Pollard, \& Dickson, 1967; Zeigler \& Leibowitz, 1957).

During the 1960s and 1970s - the period in which size constancy in children was most actively investigated - two main hypotheses were proposed to explain the difference between young children and adults in their ability to estimate the size of a distant object. Granrud (2004) has referred to these as the perceptual learning and cognitive supplementation hypotheses. According to the perceptual learning hypothesis, children are less able than adults to utilize the monocular cues, such as linear perspective, that provide information about object distance and size

This research was first presented at the 2003 meeting of the Society for Research in Child Development, held in Tampa, FL. The authors thank the ABC Child Development Center of Greeley, the University Laboratory School, the UNC Child Care Center, Robin Anthony, Pat Hano, and Jean Myer-Scott for making this research possible by helping to recruit the children who participated in the study. The authors also thank Melissa Granrud, Kathleen Cox, Julia Koc, Elianna Mondragon, Lisa Nawrot, Ryan Peterson, Eric Warneke, and Joshua Welp for their assistance in testing research participants, and Mark Alcorn, Richard Held, Terri Lewis, Bill Merriman, D. Alfred Owens, Lora Schlewitt-Haynes, Allison Sekuler, Josephine Shallo-Hoffmann, and Jeremy Wolfe for their valuable suggestions regarding the manuscript. Correspondence concerning this article may be sent to C. E. Granrud, School of Psychological Sciences, McKee 14, Campus Box 94, University of Northern Colorado, Greeley, CO 80639 (e-mail: carl.granrud@unco.edu). when distances exceed a few meters. As a result, children misperceive size at distances beyond a few meters (see, e.g., Leibowitz, 1974; Leibowitz et al., 1967). According to the cognitive supplementation hypothesis, there is no difference between children and adults in how they perceive a distant object: Both perceive the object as smaller than its actual size. However, adults estimate distant objects' sizes more accurately than young children do because they use cognitive strategies to judge size, whereas young children base their size estimates on what they perceive and do not supplement perception with cognitive strategies. As a result, young children exhibit underconstancy (i.e., they underestimate size) for distant objects (see, e.g., Granrud, 2004; Rapoport, 1967).

Empirical studies have uncovered evidence that supports both the perceptual learning (Leibowitz et al., 1967) and cognitive supplementation (Granrud, 2004; Rapoport, 1967, 1969) hypotheses. However, Shallo and Rock (1988) proposed a third explanation for the different performances of children and adults in size constancy experiments that, if correct, would imply that both earlier hypotheses are incorrect. In the present study, we tested Shallo and Rock's hypothesis.

Their hypothesis is based on Rock's idea that there are two modes of perception: the proximal mode and the constancy mode (see, e.g., Rock, 1983). The proximal mode refers to the perception of retinal image features, such as retinal size. The constancy mode refers to the perception of an object's physical properties, such as objective size. Adults can shift attention from one mode to the other. They can perceive, for example, that an object's physical size remains invariant as its distance increases (constancy mode), but they can also perceive that the object's image size is smaller when it is farther away (proximal mode). Shallo and Rock (1988) hypothesized that the proximal mode is more salient in young children than in adults and 
that the greater salience of the proximal mode in children accounts for their incomplete size constancy. According to Shallo and Rock, adults typically respond in the constancy mode and make veridical size estimates or overestimate size when they are asked to estimate the size of a distant object. Young children, however, attend to both modes and make size estimates that reflect a compromise between the object's physical size-represented in the constancy mode - and its much smaller retinal image size - represented in the proximal mode. Children, therefore, underestimate the size of a distant object.

To our knowledge, Shallo and Rock (1988) did not give their hypothesis a name. In her unpublished dissertation, Shallo (1984) hypothesized that young children exhibit underconstancy due to their "proximal mode sensitivity" (p. 11). Following Shallo's terminology, we will refer to this explanation for children's performance in size constancy tasks as the proximal mode sensitivity hypothesis.

To test the proximal mode sensitivity hypothesis, Shallo and Rock (1988) presented a standard object at a distance of $61 \mathrm{~m}$ and asked participants to indicate which of nine nearby comparison objects matched the standard object in size. When the comparison objects were equidistant from the participant - and therefore subtended different visual angles - 5- to 8-year-old children exhibited underconstancy: They chose a comparison object smaller than the standard object. But when the comparison objects were placed at varying distances and subtended equal visual angles, 5- to 8-year-old children exhibited nearly accurate size constancy. According to Shallo and Rock, when the comparison objects have equal retinal image sizes, participants cannot select a match for the standard object on the basis of proximal mode information because every comparison object is equally similar to the standard object in retinal size; therefore, they are forced to respond in the constancy mode. On the basis of these findings, Shallo and Rock concluded that children of ages 5-8 are capable of veridical size perception in the constancy mode but that this ability is apparent only when proximal mode information is neutralized and participants are forced to respond in the constancy mode. Shallo and Rock further concluded that previous studies did not reveal 5- to 8-yearold children's size constancy abilities because the methods used in those studies allowed children to attend to both modes of perception.

Shallo and Rock's (1988) results appear to confirm the proximal mode sensitivity hypothesis. However, other studies have supported the cognitive supplementation hypothesis (Granrud, 2004; Rapoport, 1967, 1969). For example, Granrud reported that, at any given age from 5 to 10 years, children who exhibit size constancy or overconstancy (i.e., overestimation of size) for a distant object frequently describe explicit strategies that they use to infer the object's size, whereas children who exhibit underconstancy generally report that they respond on the basis of how an object "looks" when estimating its size. The proximal mode sensitivity and cognitive supplementation hypotheses appear to be incompatible. The former proposes that accurate size constancy for distant objects is a feature of perception (in the constancy mode), whereas the latter proposes that size constancy for distant objects is not a feature of perception but instead results from a deliberate cognitive judgment. Findings that support the proximal mode sensitivity hypothesis and those that support the cognitive supplementation hypothesis therefore seem difficult to reconcile.

The proximal mode sensitivity hypothesis also conflicts with the perceptual learning hypothesis. The latter hypothesis proposes that young children are incapable of accurate size constancy for distant objects due to their relative insensitivity to the monocular cues - such as linear perspective - that provide information on object distance and size at far distances (see, e.g., Leibowitz, 1974). If this view is correct, young children should be unable to perceive size accurately in the constancy mode when distances exceed a few meters. Shallo and Rock's (1988) findings, which support the proximal mode sensitivity hypothesis, therefore conflict with findings by Leibowitz et al. (1967), which appear to support the perceptual learning hypothesis (these findings are described below, in the General Discussion section).

Due to these conflicting findings in the literature, it is not yet clear what causes age-related changes in size constancy performance during childhood, and additional tests of the perceptual learning, cognitive supplementation, and proximal mode sensitivity hypotheses are needed. The present study was part of a research program designed to identify the causes of developmental changes in size constancy performance by conducting systematic tests of these three hypotheses. Here, we test the proximal mode sensitivity hypothesis by attempting to replicate Shallo and Rock's (1988) finding that 5- to 6-year-old children exhibit size constancy for a distant object when tested with comparison objects that are matched for retinal size.

\section{EXPERIMENT 1}

Shallo and Rock (1988) reported three experiments. Their first experiment replicated Brislin and Leibowitz's (1970) finding that 5- to 8-year-old children underestimate the size of a standard object at distances of 30.5 and $61 \mathrm{~m}$ when they estimate size by choosing from a set of sequentially presented comparison objects that vary in retinal size. In Shallo and Rock's third experiment, 5- to 6-year-olds underestimated the size of a standard object at $61 \mathrm{~m}$ when the comparison objects had varying retinal sizes and were presented simultaneously. In their second experiment, in which they tested the proximal mode sensitivity hypothesis, the comparison objects were presented simultaneously at varying distances and subtended equal visual angles. They found that 5- to 8-year-old children exhibited nearly accurate size constancy for a standard object at a distance of $61 \mathrm{~m}$.

The main goal of the present study was to replicate the findings of Shallo and Rock's (1988) second experiment. Participants viewed one circular standard object that was directly in front of them at a distance of $61 \mathrm{~m}$. Nine nearby comparison objects were positioned to the participant's 
right. One of the comparison objects matched the standard object in size. The participant's task was to point to the comparison object that was the same size as the standard object.

Each participant was tested in one of two conditions. In the matched retinal size condition, the comparison objects were arranged in an arc with each object at a different distance $(0.91-4.57 \mathrm{~m})$ from the participant (see Figure 1). The nine comparison objects subtended equal visual angles. This condition was designed to replicate that of Shallo and Rock's (1988) second experiment. In the variable retinal size condition, the nine comparison objects were arranged in a straight line parallel to the participant's line of sight when he or she was viewing the standard object. In this condition, the comparison objects subtended different visual angles.

The variable retinal size condition did not replicate any condition of Shallo and Rock's (1988) experiments. In their third experiment, the comparison objects were all presented at the same distance from the research participant $(4.57 \mathrm{~m})$, whereas in the present experiment we placed the comparison objects at varying distances from the participant in the variable retinal size condition. Since Shallo and Rock gave little information regarding the arrangement of the comparison objects in their third experiment, it did not seem feasible to replicate exactly the conditions of that experiment. Instead, the variable retinal size condition was designed to test participants under conditions similar to the matched retinal size condition, but with comparison objects that had varying retinal sizes. If the proximal mode sensitivity hypothesis were correct, 5- to 6-year-old children would be expected to exhibit size constancy in the matched retinal size condition and underconstancy in the variable retinal size condition, since proximal mode information was not neutralized in the latter condition.

Our experiment also included a pretest in which participants viewed three standard objects, one at a time, from a distance of $2.74 \mathrm{~m}$. In the pretest, we used the same procedure and comparison objects as we did in the test trial. The pretest was administered to ensure that the participants understood the task and could accurately identify a comparison object that matched a standard object when the standard object was nearby and its size was clearly perceptible. This pretest procedure differed from that used by Shallo and Rock (1988). In their pretest, children viewed a black sheet - at a distance of $0.9 \mathrm{~m}$ - onto which six white circles were affixed, two of which were equal in size. The children were asked to pick the two circles that were equal in size. Because Shallo and Rock used different stimuli and instructions in their pretest than they did in their test trial, it seemed possible that a child could pass the pre-

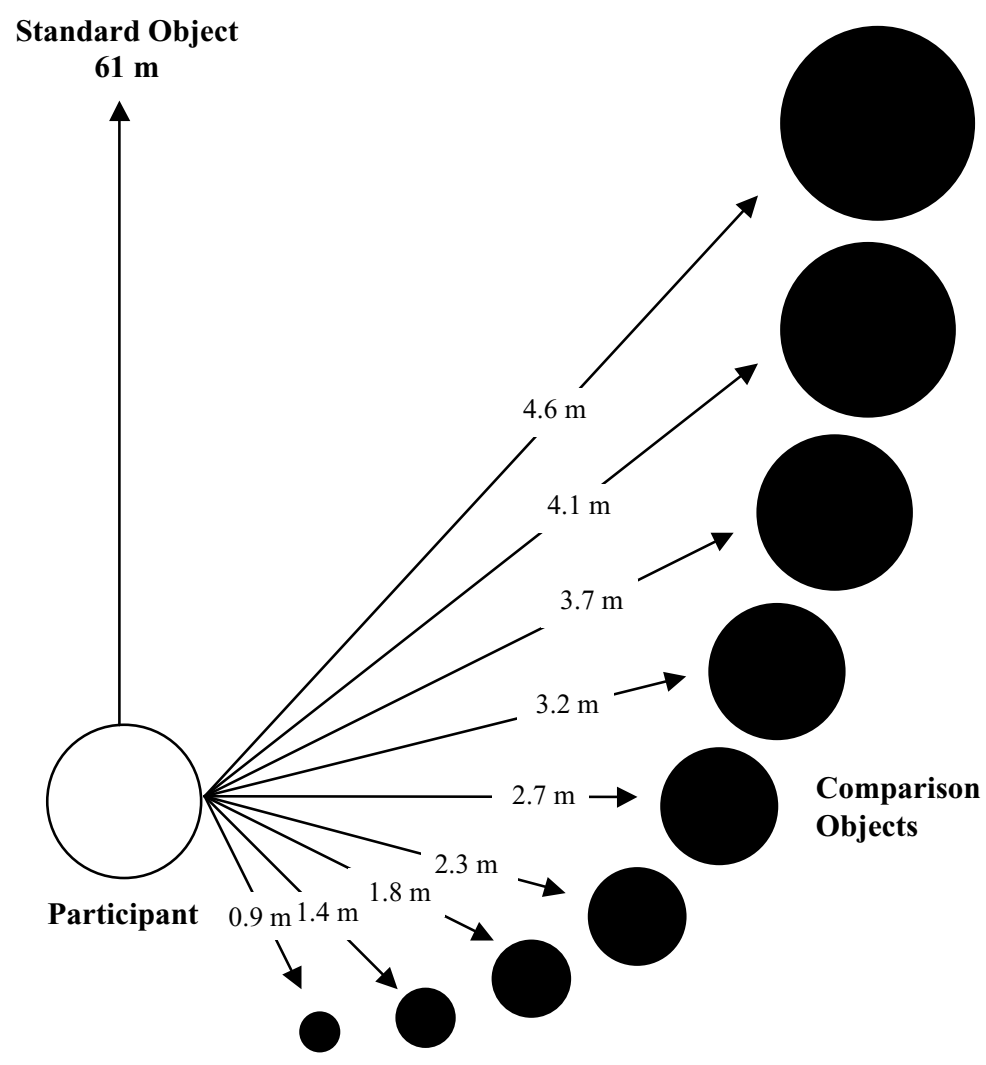

Figure 1. Approximate arrangement of comparison objects in the matched retinal size condition. Diagram not drawn to scale. Exact object sizes and distances given in text. 
test but be unable to follow the instructions or accurately point to comparison objects up to $4.57 \mathrm{~m}$ away in the test trial. Such a child might exhibit a response bias or random responses in the test trial. If this occurred, children's responses in Shallo and Rock's test trial may not have provided a valid measure of the children's perception of the standard object's size. Since we used the same comparison objects and instructions in the pretest of Experiment 1 of the present study as we did in the test trial, it was clear that children who passed the pretest understood the task and could point accurately to the comparison object that matched a standard object in size.

\section{Method}

Participants. Twenty participants were tested in each of four age groups: 5 -year-olds ( 11 males and 9 females; mean age $=5$ years, 1 month; age range: 4 years, 6 months to 5 years, 6 months), 6 -yearolds ( 9 males and 11 females; mean age $=5$ years, 11 months; age range: 5 years, 7 months to 6 years, 4 months), 9-year-olds ( 9 males and 11 females; mean age $=8$ years, 11 months; age range: 8 years, 2 months to 9 years, 5 months), and adults ( 5 males and 15 females; mean age $=23$ years, 8 months; age range: 19 years, 7 months to 31 years, 8 months). An additional nine 5-year-olds, four 6-yearolds, two 9-year-olds, and one adult were tested but excluded from the sample because they failed the pretest. (The criteria for passing the pretest are described below.)

The children who participated in the study were recruited from a university child care center, a university laboratory school, and a local daycare center. The adult participants were students in an undergraduate psychology class who volunteered for the study to earn extra credit and gave written informed consent before participating. Written informed consent was obtained from the parents of the children who participated, and verbal assent was obtained from the children. All research participants were treated in accordance with the ethical guidelines of the American Psychological Association and the Society for Research in Child Development.

Apparatus. The research participants were tested on sunny days on a grass-covered playground that was adjacent to the daycare center or to the university laboratory school. Testing was conducted when the sun was overhead or at the participant's back.

The stimulus objects were designed to replicate those used in the second experiment of Shallo and Rock's (1988) study. Shallo (1984) described the stimuli in greater detail than did Shallo and Rock. The objects used in the present study matched the English units of size and distance reported by Shallo. The metric values reported by Shallo and Rock and in this article represent values converted from English units and are therefore approximations.

For the test trial, the standard object was a white circular disk, $61 \mathrm{~cm} \mathrm{(24} \mathrm{in.)} \mathrm{in} \mathrm{diameter,} \mathrm{made} \mathrm{of} \mathrm{1-cm-thick} \mathrm{foam-core} \mathrm{board.}$ The standard object was positioned at a distance of $61 \mathrm{~m}(200 \mathrm{ft})$ from the participant and subtended $0.57^{\circ}$ of visual angle. The standard object was positioned so that all other objects on the playground (e.g., trees and fences) were at least $20 \mathrm{~m}$ from the standard object.

The comparison objects consisted of nine white circular disks, $76.2,68.6,61.0,53.3,45.7,38.1,30.5,22.9$, and $15.2 \mathrm{~cm}$ in diameter (i.e., 30, 27, 24, 21, 18, 15, 12, 9, and 6 in. in diameter), made of 1-cm-thick foam-core board. The standard object and each comparison object were permanently mounted on wooden supports, painted flat black, with plastic tubing (not visible to the participant) affixed to the back of the supports. The supports were held upright by metal stakes inserted into the tubing and driven into the ground. Each wooden support had a width equal to one sixth of the diameter of the comparison object that it supported. The center of each object (standard and comparison) was $122 \mathrm{~cm}$ from the ground.

The nine comparison objects were presented simultaneously to the participant's right, with a $90^{\circ}$ separation between the standard object and the object at the center of the comparison object array. The comparison objects were arranged in size order, with the largest object on the left and the smallest object on the right, from the participant's viewpoint. Each object was separated from its neighbors by approximately $2^{\circ}$ of visual angle.

In the matched retinal size condition, the comparison objects were arranged in an arc with the 76.2-cm object at a distance of $4.57 \mathrm{~m}(15 \mathrm{ft})$, the $68.6-\mathrm{cm}$ object at $4.11 \mathrm{~m}(13.5 \mathrm{ft})$, the $61.0-\mathrm{cm}$

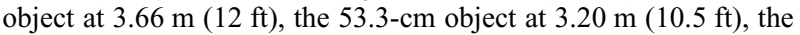
$45.7-\mathrm{cm}$ object at $2.74 \mathrm{~m}(9 \mathrm{ft})$, the $38.1-\mathrm{cm}$ object at $2.29 \mathrm{~m}(7.5 \mathrm{ft})$, the $30.5-\mathrm{cm}$ object at $1.83 \mathrm{~m}(6 \mathrm{ft})$, the $22.9-\mathrm{cm}$ object at $1.37 \mathrm{~m}$

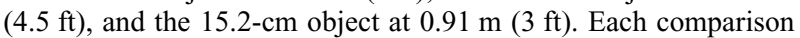
object subtended the same visual angle: $9.46^{\circ}$. Figure 1 illustrates the arrangement of the comparison objects in this condition.

In the variable retinal size condition, the comparison objects were arranged in a straight line, parallel to the participant's line of sight when he or she was viewing the standard object and perpendicular to the participant's line of sight when he or she was viewing the object at the center of the comparison object array. The object at the center of the array was positioned at a distance of $2.74 \mathrm{~m}(9 \mathrm{ft})$ from the participant. The comparison objects subtended visual angles ranging from $2.74^{\circ}$ to $10.90^{\circ}$ in this condition.

The same comparison objects were used for the test trial and the pretest. In the pretest, we used nine standard objects that matched the nine comparison objects in size. Each participant viewed three standard objects (chosen from the nine possible standard objects) one at a time. Each standard object was presented directly in front of the participant at a distance of $2.74 \mathrm{~m}$. There was a $90^{\circ}$ separation between the standard object and the object at the center of the comparison object array. The standard objects were temporarily attached with Velcro to a black wooden support, $10.16 \mathrm{~cm}$ in width, with the center of the object $122 \mathrm{~cm}$ from the ground.

To control for viewing height, the 5- and 6-year-old children stood on a $30.5-\mathrm{cm}$-high Styrofoam platform while viewing the stimulus objects, the 9-year-old children stood directly on the ground, and the adult participants viewed the objects through viewing windows. As a result, for all pretest and test trials, the participants viewed the stimulus objects from an eye height of approximately $122 \mathrm{~cm}$. The viewing windows were cut in two adjacent black cardboard walls (each $1.83 \mathrm{~m}$ wide and $1.83 \mathrm{~m}$ high) that formed a $90^{\circ}$ angle. One wall was directly in front of the participant and the other was to the right when the participant was viewing the standard object. The standard and comparison objects were viewed through equal-sized windows $-76 \mathrm{~cm}$ wide and $12 \mathrm{~cm}$ high - cut into the two walls. The center of each viewing window was $122 \mathrm{~cm}$ above the ground. All the comparison objects were visible simultaneously through the viewing window. The viewing platform and viewing window were designed to approximate those used by Shallo and Rock (1988).

Procedure. There were two conditions in the experiment: the matched retinal size condition and the variable retinal size condition. The experiment had a between-subjects design: Each participant was tested in one condition. Half the participants in each age group were tested in each condition. The condition assigned to the first participant in each age group was randomly chosen. The first 5 participants in the age group were tested in this condition. The next 5 participants were tested in the other condition. This pattern was continued until all 20 participants in each age group had been tested.

The experiment consisted of three pretest trials and one test trial. In the pretest trials, three different standard objects were presented one at a time. For each participant, these objects were chosen from the nine possible standard objects using the following procedure: One standard object was chosen randomly from the three largest objects $(76.2,68.6$, and $61 \mathrm{~cm})$, one was chosen randomly from the three intermediate-sized objects $(53.3,45.7$, and $38.1 \mathrm{~cm})$, and one was chosen randomly from the three smallest objects $(30.5,22.9$, and $15.2 \mathrm{~cm})$. The participants viewed the same comparison object array (matched or variable retinal size) in the pretest that they viewed in the test trial. Before each pretest trial, the participant faced in the 
direction opposite that of the standard object while the standard object was put into place. When the standard object was in place, the participant was instructed to turn around and view the standard object. In each pretest trial, the participant was instructed to point to the comparison object that was the same size as the standard object. If either the data or the reliability experimenter (the roles of these two experimenters are described below) was unable to determine where the participant was pointing, the participant was asked to repeat his or her response. If either experimenter was still uncertain, the participant was asked to walk to and touch the comparison object that was the same size as the standard object. No time limit was placed on the participant. The pretest was given to ensure that participants understood the task and could respond accurately when the standard object was nearby and its size was therefore clearly perceptible. Participants were included in the sample if they were correct on at least two of the three pretest trials, and if they made one error, that the incorrect comparison object that they chose was directly adjacent to the correct object in the comparison object array.

After the pretest was completed, the participant was instructed to face in the direction opposite that of the standard object while the pretest standard object was removed and the test trial standard object was put in place. When the standard object was in place, the participant was instructed to turn around and view the standard object. The participant was then instructed to point to the comparison object that was the same size as the standard object. If an experimenter could not determine where the participant was pointing, the procedure that was used in the pretest (described above) was followed. No time limit was placed on the participant. The dependent variable for each participant was the diameter of the comparison object that was chosen as matching the standard object in size. This was referred to as the participant's size estimate.

Three experimenters conducted the study. Prior to the start of the study, one experimenter was designated as the data experimenter and another was designated as the reliability experimenter. These experimenters stood behind the participant during the pretest and test trial and independently recorded the participant's responses. The data recorded by the data experimenter were used in all the analyses. Pearson's $r$ correlation coefficients were computed to measure agreement between the data experimenter and the reliability experimenter. These analyses revealed correlations of $r=.99$ for the pretest trials $(n=240)$ and $r=.98$ for the test trials $(n=80)$, which indicates that there was a very high level of agreement between the experimenters.

The data observer interacted with the research participants and gave them instructions. To control for experimenter bias, the data experimenter was kept unaware of the study's hypotheses throughout the study. In addition, the data experimenter was instructed not to inspect the test trial standard object and throughout the study was kept unaware of the standard object's size and of which comparison object matched the standard object in size. The third experimenter put the standard objects in place.

\section{Results}

Table 1 shows each age group's mean size estimate in each condition and the overall mean for each age group (irrespective of condition). These means were compared in a two-factor between-subjects ANOVA with condition (matched retinal size and variable retinal size) and age ( 5 years, 6 years, 9 years, and adults) as factors. This analysis revealed a significant main effect of age $[F(3,72)=$ $10.06, p<.05]$, no significant main effect of condition $[F(1,72)=0.43, p>.05]$, and no significant age $\times$ condition interaction $[F(3,72)=0.45, p>.05]$. The significant main effect of age indicates that size estimates varied significantly as a function of age. The lack of a significant main effect of condition indicates that size estimates did
Table 1

Mean Estimated Size of 61-cm Standard Object in Each Condition and Overall Mean for Each Age Group

\begin{tabular}{|c|c|c|c|c|c|c|}
\hline \multirow[b]{3}{*}{ Age } & \multicolumn{4}{|c|}{ Condition } & & \\
\hline & \multicolumn{2}{|c|}{$\begin{array}{c}\text { Matched } \\
\text { Retinal Size* }\end{array}$} & \multicolumn{2}{|c|}{$\begin{array}{c}\text { Variable } \\
\text { Retinal Size* }\end{array}$} & \multicolumn{2}{|c|}{ Overall $^{\dagger}$} \\
\hline & $M$ & $S D$ & $M$ & $S D$ & $M$ & $S D$ \\
\hline 5 years & 44.95 & 11.62 & 45.72 & 12.44 & 45.34 & 11.72 \\
\hline 6 years & 51.07 & 11.36 & 50.29 & 8.16 & 50.68 & 9.64 \\
\hline 9 years & 57.15 & 8.98 & 63.25 & 11.39 & 60.20 & 10.46 \\
\hline Adult & 60.20 & 9.80 & 60.20 & 8.39 & 60.20 & 8.88 \\
\hline
\end{tabular}

${ }^{*} n=10$ for all age groups. ${ }^{\dagger} n=20$ for all age groups.

not differ significantly between the matched and variable retinal size conditions. The finding of no significant interaction indicates that condition (whether or not the comparison objects were matched in retinal size) had no effect on size estimates in any age group.

Tukey follow-up tests were conducted to compare the overall means of the four age groups. An $\alpha$ level of .05 was used, and HSD equaled 8.49. These tests revealed that the means for the 5- and 6-year-old groups were significantly lower than the means for the 9-year-old and adult groups. The mean for the 5-year-old group did not differ significantly from the mean for the 6-year-old group, and the mean for 9-year-old group did not differ significantly from the mean for the adult group.

The graphs in Figure 2 show the number of participants who chose each comparison object as a size match for the 61-cm standard object in the matched (panel A) and variable (panel B) retinal size conditions. The 5- and 6-year-old groups were combined, as were the 9-year-old and adult groups, to make the graphs more easily comprehensible. As can be seen in the graphs, the majority of the 5- and 6-yearolds made underconstancy responses in both conditions (16/20 in the matched retinal size condition and 17/20 in the variable retinal size condition), whereas the majority of the 9-year-olds and of the adults made constancy or overconstancy responses in both conditions (11/20 and 15/20 in the matched and variable retinal size conditions, respectively).

\section{Discussion}

Shallo and Rock (1988) reported that children as young as 5 years of age exhibited nearly accurate size constancy for a standard object that was $61 \mathrm{~m}$ away when they chose a size match from comparison objects that were matched for retinal size. In our first experiment, matching the comparison objects for retinal size had no effect on the children's size estimates: The 5- and 6-year-olds underestimated the size of the standard object in both the matched and variable retinal size conditions. These findings do not support Shallo and Rock's conclusion that 5-year-old children accurately perceive the sizes of distant objects when they attend to the constancy mode. Instead, our findings support the conclusion drawn in several previous studies that 5- to 6-year-old children underestimate the sizes of distant objects (Brislin \& Leibowitz, 1970; Leibowitz et al., 1967; Zeigler \& Leibowitz, 1957). 
A Matched Retinal Size Condition

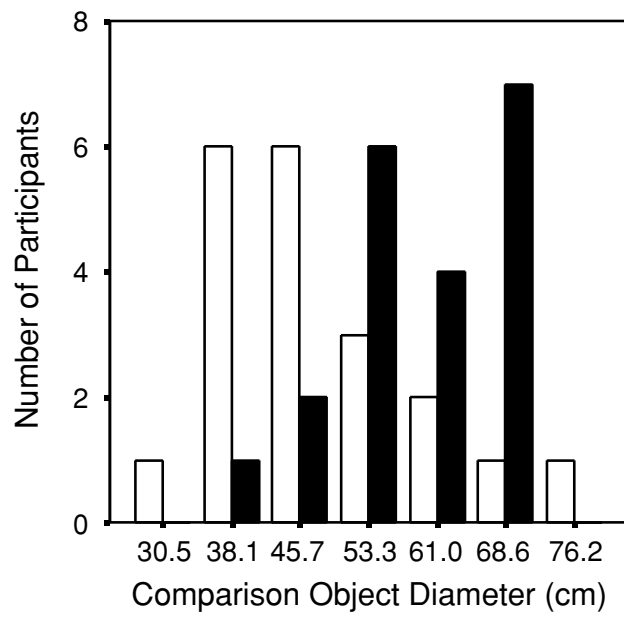

B

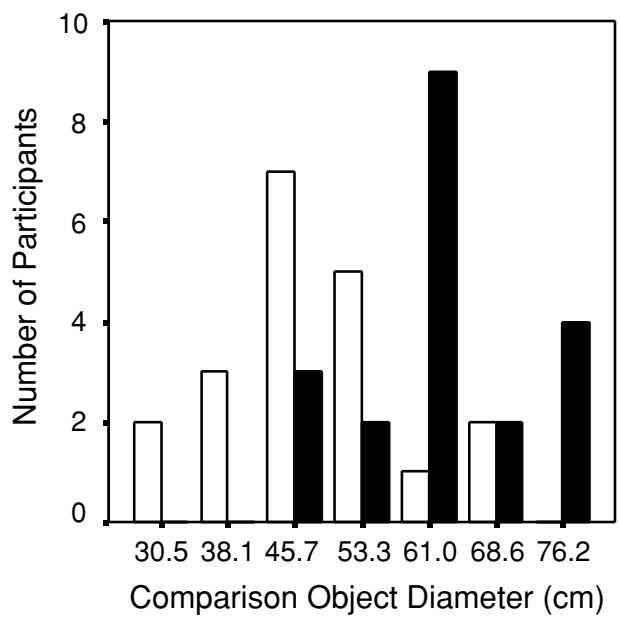

Age Group

5 and 6 years

9 years and adults
Age Group

$\square 5$ and 6 years

9 years and adults

Figure 2. Number of participants in each age group who chose each comparison object as a size match for the 61-cm standard object in the matched retinal size condition (A) and the variable retinal size condition (B) in Experiment 1.

The sample sizes were relatively small in Experiment 1: We tested only 10 participants in each condition within each age group. However, this does not appear to threaten the validity of the conclusions drawn above. The findings that the 5- and 6-year-olds' size estimates differed significantly from the accurate value of $61 \mathrm{~cm}$ in each condition of the experiment indicate that the statistical power was adequate to establish that these children underestimated size when the comparison objects were matched in retinal size and when they varied in retinal size.

As noted above, in the variable retinal size condition we used a different comparison object arrangement than that used by Shallo and Rock (1988) in their third experiment, in which the comparison objects were presented at one distance $(4.57 \mathrm{~m})$ and had varying retinal sizes. However, the young children's results in the variable retinal size condition were similar to those obtained by Shallo and Rock in their third experiment. The mean size estimate by 5 - to 6-year-old children in Shallo and Rock's third experiment was $42.7 \mathrm{~cm}$, in comparison with the 5-year-olds' mean of $45.7 \mathrm{~cm}$ in the variable retinal size condition of our first experiment. These similar results indicate that underconstancy in young children is a reliable effect when size estimates are measured using comparison objects that vary in retinal image size, and that this finding does not depend on the exact arrangement of the comparison objects. Furthermore, the similar results of these two experiments allow us to rule out the possibility that unusual results in the variable retinal size condition caused the finding of no difference between the young children's means in the matched and variable retinal size conditions of Experiment 1.

Unlike the 5- and 6-year-olds' mean size estimates, those of the 9-year-olds were close to the accurate value of $61 \mathrm{~cm}$ in both conditions of the experiment. Previous 
research had not clearly established whether 9-year-old children exhibit size constancy for distant objects. In the Leibowitz et al. (1967) study, 91/2-year-olds exhibited nearly accurate size constancy at 30.5 and $61 \mathrm{~m}$ under binocular viewing conditions but slight underconstancy under monocular conditions. In the Brislin and Leibowitz (1970) study, 9-year-olds exhibited a small degree of underconstancy at 30.5 and $61 \mathrm{~m}$. However, it was not reported in either study whether the 9 - or $91 / 2$-year-olds' size estimates differed significantly from the accurate size values or from the size estimates made by adults. Although additional research will be needed to determine whether 9-year-old children have any size constancy deficits relative to adults, the results of Experiment 1 provide evidence that 9-year-old children are capable of adult levels of size constancy at distances up to $61 \mathrm{~m}$ and suggest that the varying results obtained with this age group in the Leibowitz et al. and Brislin and Leibowitz studies resulted from random variation among sample means.

The pretest in Experiment 1 was necessary to validate the method used to measure children's size estimates. As mentioned above, Shallo and Rock (1988) did not use in their pretest the same stimulus objects or instructions that they used in their test trial. Since no children were excluded from the Shallo and Rock study on the basis of their pretest performance (whereas 31\% of the 5-year-olds and $17 \%$ of the 6 -year-olds tested in our Experiment 1 failed the pretest), it seems possible that Shallo and Rock's sample included some children who did not understand the test trial task or instructions. In Experiment 1, we used the same procedures and comparison objects in both the pretest and the test trial. Every participant included in the sample had passed the pretest and therefore had demonstrated that he or she understood the instructions and could point accurately to comparison objects that matched standard objects in size. Furthermore, to pass the pretest participants had to point accurately to comparison objects from the left side, middle, and right side of the comparison object array (because the three pretest standard objects matched comparison objects from each of these areas). Children who could do this were clearly not exhibiting a response bias, such as pointing toward the middle of the comparison object array or consistently choosing the larger comparison objects. This suggests that these children's test trial performance provided a valid measure of their size estimates for the standard object.

The results of a study by Granrud and Morreale (2001) provide additional evidence that the children did not exhibit a response bias in our test trial in Experiment 1. Each child in the Granrud and Morreale study viewed one of three different-sized standard objects at a distance of $61 \mathrm{~m}$ and estimated the standard object's size by choosing a size match from a set of comparison objects that was the same as the one we used in the variable retinal size condition in Experiment 1. Children in the 5- and 6-yearold groups consistently exhibited underconstancy. In addition, their size estimates varied significantly as a function of the standard object's size (i.e., they made larger size estimates when the standard object was larger and smaller size estimates when the object was smaller). This finding suggests that the children were not exhibiting a response bias, such as choosing the object at the center of the comparison object array, but were instead responding on the basis of the perceived size of the standard object. Since we used the same comparison objects and similar procedures that Granrud and Morreale used, it seems unlikely-given Granrud and Morreale's results - that children in our study exhibited a response bias in the test trial of Experiment 1.

\section{EXPERIMENT 2}

As noted above, we used a different pretest in Experiment 1 than did Shallo and Rock (1988). Since no other substantive difference between the matched retinal size condition of Experiment 1 and Shallo and Rock's second experiment is apparent, it seemed possible that the different pretest procedures used in the two experiments caused the different test trial results. As was mentioned earlier, it is possible that Shallo and Rock's pretest was unable to identify children who did not understand the test trial instructions, whereas such children were most likely excluded from Experiment 1 of the present study on the basis of the pretest. It is also possible that the extensive pretest procedure we used in Experiment 1 caused the younger children to become fatigued and therefore prevented them from exhibiting their best possible performance in the test trial. We designed Experiment 2 to explore these possibilities. In Experiment 2, we used the same pretest and test trial procedures that Shallo and Rock used in their second experiment. The goal of our second experiment was to determine whether 6-year-old children would exhibit underconstancy in the matched retinal size condition-as they did in Experiment 1-or size constancy - as they did in Shallo and Rock's second experiment-when Shallo and Rock's pretest and test trial procedures were followed as precisely as possible.

We tested the participants in Experiment 2 only in the matched retinal size condition. The results of Experiment 1 and those of Shallo and Rock's (1988) third experiment were similar when children were tested with comparison objects that varied in retinal size: Children in the 5- and 6-year-old groups exhibited significant underconstancy. This finding appears to be reliable; thus, replicating the results from the variable retinal size condition of Experiment 1 seemed unnecessary. Sixteen 6-year-old children and 16 college-aged adults were tested in Experiment 2. No 5-year-old children were available to participate, so this age group was not represented in Experiment 2.

\section{Method}

Participants. Sixteen 6-year-old children ( 8 males and 8 females; mean age $=5$ years, 11 months; age range: 5 years, 6 months to 6 years, 6 months) and 16 adults ( 3 males and 13 females; mean age $=19$ years, 5 months; age range: 18 years, 0 months to 21 years, 2 months) participated in the study. No participants were excluded from the sample on the basis of their pretest performance. The children were recruited from a summer program for school-aged children administered by a local daycare center. The adults, students 
in an undergraduate psychology class, participated in the study for extra credit. As in Experiment 1, we obtained informed consent from adult participants and from a parent of each child who participated, and we obtained verbal assent from the children who participated. All the research participants were treated in accordance with the ethical guidelines of the American Psychological Association and the Society for Research in Child Development.

Apparatus. The test trial apparatus was identical to the one that we used in the matched retinal size condition of Experiment 1. The display that we used in the pretest was a sheet of stiff black construction paper $71 \mathrm{~cm}$ wide and $56 \mathrm{~cm}$ high onto which six white circles were glued in a quasi-random pattern. Two circles had the same diameter $-17.8 \mathrm{~cm}$. The other four circles had diameters of 2.5, 7.6, 12.7 , and $22.9 \mathrm{~cm}$. Shallo and Rock (1988) gave little information regarding their pretest display. Shallo (1984) provided slightly more information and described it in the following way: "The pre-test consisted of a black cardboard chart with six white circles on it. Only two of the circles were of equal size" (p. 42). She also noted that an experimenter held the display at a distance of approximately $0.9 \mathrm{~m}$ from the child, at a height of approximately $1.2 \mathrm{~m}$. The pretest display that we used in our Experiment 2 was designed to approximate Shallo and Rock's display as closely as possible, given the limited information that they provided.

Procedure. For the children, the experiment consisted of a pretest and one test trial. The adult participants were given the test trial only. The test trial followed the same procedure that we used in Experiment 1, with one exception: Participants were tested in the matched retinal size condition only.

Two experimenters conducted the pretest indoors. Experimenter 1 held the pretest display at a distance of approximately $0.9 \mathrm{~m}$ in front of the child, who stood facing the display. The child received the following instructions: "Two of these circles are the same size. Point to the circles that are the same size." This procedure approximated that used by Shallo and Rock (1988), described by Shallo (1984) in the following way: "Observers were asked to pick the circles that were the same size" (p. 42). Both experimenters recorded the child's response. A child was included in the sample if both experimenters scored his or her response as correct. All the children tested in Experiment 2 answered the pretest question correctly (according to both experimenters); thus, no child was excluded from the sample. When the pretest was completed, the experimenters and the child walked outdoors and the test trial was conducted.
As in Experiment 1, we computed a Pearson's $r$ correlation to measure agreement between the data experimenter and the reliability experimenter in the test trial. A correlation of $r=.99(N=32)$ was found, indicating a high degree of agreement between the experimenters.

\section{Results}

The adult group's mean size estimate was $62.87 \mathrm{~cm}$ $(S D=6.53)$. The children's mean size estimate was $50.01 \mathrm{~cm}(S D=10.02)$. An independent samples $t$ test indicated that these means differed significantly $[t(30)=$ $4.30, p<.05]$. Planned one-sample $t$ tests were also conducted to compare each group's mean to the accurate value of $61 \mathrm{~cm}$. The adults' mean size estimate did not differ significantly from the accurate value of $61 \mathrm{~cm}[t(15)=1.14$, $p>.05]$. The children's mean differed significantly from $61 \mathrm{~cm}[t(15)=-4.39, p<.05]$, indicating that the children significantly underestimated the standard object's size.

Figure 3 shows the number of participants in each age group who chose each comparison object as a size match for the 61-cm standard object. As can be seen in the figure, the majority of 6-year-olds made underconstancy responses (13/16), whereas the majority of adults made constancy or overconstancy responses $(14 / 16)$.

\section{Discussion}

The children in Experiment 2 underestimated the size of the standard object, whereas the adults exhibited nearly accurate size constancy. These findings suggest that the different results in the matched retinal size condition of Experiment 1 and in Shallo and Rock's (1988) second experiment cannot be attributed to the use of different pretest procedures. Our finding that 6-year-old children exhibit underconstancy for a distant object when comparison objects are matched in retinal size appears to be a reliable effect, despite our use of Shallo and Rock's pretest procedure.

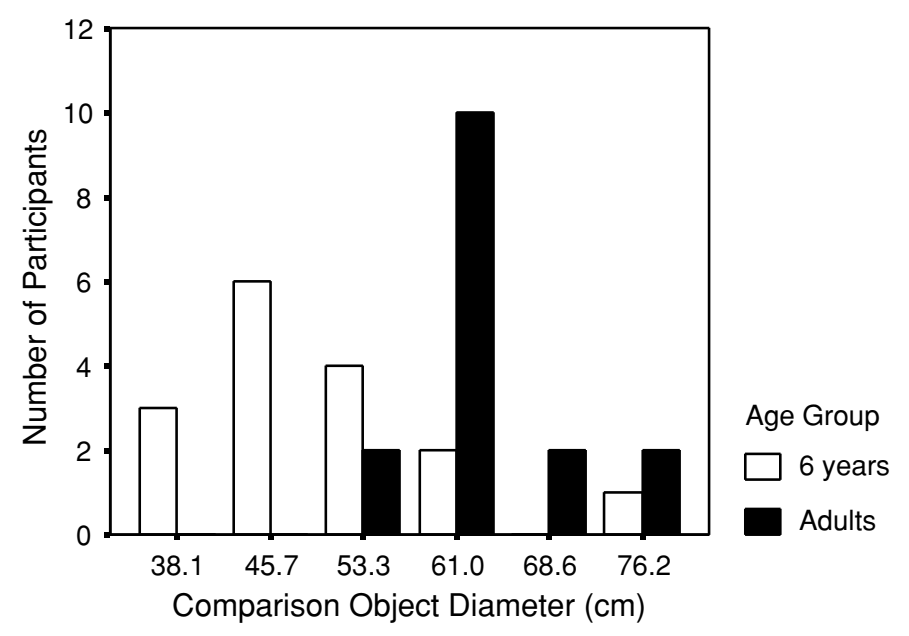

Figure 3. Number of participants in each age group who chose each comparison object as a size match for the $61-\mathrm{cm}$ standard object in Experiment 2. 
Four of the twenty-four 6-year-olds that we tested in Experiment 1 failed the pretest, and we excluded them from the sample. In Experiment 2, we excluded no children on the basis of their pretest performances. It is likely that some children whom we included in Experiment 2 would have failed our Experiment 1 pretest. The pretest was an important part of Experiment 1, necessary to ensure that the test trial provided a valid measure of children's size estimates. However, the results of Experiment 2 suggest that the finding of underconstancy in 6-year-old children does not depend on the exclusion of children who are unable to pass the Experiment 1 pretest.

\section{GENERAL DISCUSSION}

This study did not replicate Shallo and Rock's (1988) main finding. They reported that children as young as 5 years of age showed nearly accurate size constancy when choosing a match for a distant object from a set of comparison objects that were matched for retinal size. In the present study, 5- to 6-year-old children underestimated the size of the standard object in the matched retinal size condition in both experiments.

Our findings do not support Shallo and Rock's (1988) conclusions that young children are capable of veridical size constancy when attending to the constancy mode and that young children's underconstancy, which was found in previous studies, resulted from a compromise between the proximal and constancy modes. To the contrary, our results suggest that children perceive a distant object as smaller than its actual size even when they attend to the constancy mode. As Shallo and Rock pointed out, when the comparison objects are matched in retinal size, participants cannot select a comparison object that matches the standard object on the basis of proximal mode information because all the comparison objects are equally similar to the standard object in retinal image size. As a result, participants are forced to attend to the constancy mode. Our findings in Experiments 1 and 2 that 5- and 6-yearolds exhibit underconstancy when comparison objects are matched for retinal size therefore suggest that children at these ages perceive a distant object as significantly smaller than its actual size when they are attending to the objective size of the object. The results of this study support the conclusion drawn in earlier studies that 5- and 6-year-old children do not exhibit complete size constancy for distant objects (Brislin \& Leibowitz, 1970; Leibowitz et al., 1967; Zeigler \& Leibowitz, 1957).

We do not know why the present study and the Shallo and Rock (1988) study yielded different results. It is possible that subtle differences between the studies caused their different results. Future research designed to identify the conditions that are necessary for reproducing Shallo and Rock's results would be worthwhile. However, given the results of the present study, the finding that children as young as 5 years of age exhibit size constancy for a distant object seems elusive at best. In contrast, the finding that 5- and 6-year-old children underestimate the size of a distant object is a robust effect that has been obtained in numerous studies using a variety of experimental methods. Children at these ages have exhibited underconstancy at viewing distances of 30.5-61 m when stimulus objects are viewed under monocular and binocular conditions (Leibowitz et al., 1967), when comparison objects are presented sequentially (Brislin \& Leibowitz, 1970; Leibowitz et al., 1967) and simultaneously (present study), when comparison objects are separated from the standard object by large and small angles (Brislin \& Leibowitz, 1970), when comparison objects have varying and matched retinal sizes (present study), when a variable comparison object is adjusted to match the size of a standard object (Zeigler \& Leibowitz, 1957), and when varying instructions are given (Granrud, 2004).

It is possible that future research will confirm Shallo and Rock's (1988) findings. Given this study's results, however, the proximal mode sensitivity hypothesis is not well supported by the evidence that currently exists. The cognitive supplementation hypothesis, on the other hand, has recently received strong empirical support. In a study reported by Granrud (2004), 5- to 10-year-old children participated in a size constancy task and a test that assessed their knowledge of the effects of distance on image size-referred to as the perceptual knowledge test. Regardless of age, children who scored above the median on the perceptual knowledge test generally exhibited size constancy or overconstancy for a distant object (61 m away), whereas children who scored below the median typically underestimated size. When asked to justify their size estimates, children who exhibited constancy or overconstancy (generally the high scorers) frequently described deliberate strategies that they used to judge the distant object's size, whereas children who exhibited underconstancy (generally the low scorers) typically reported that they based their size estimates on how the object "looked." In a second study reported by Granrud, high scorers responded differently when instructed to estimate a distant object's actual size than when instructed to estimate an object's apparent size (the size that an object "looks"); low scorers, on the other hand, made similar size estimates in response to these two types of instructions. When the children estimated the size of a nearby object ( $6 \mathrm{~m}$ away), their perceptual knowledge score did not correlate with their size estimation accuracy. This finding suggests that the high and low scorers did not differ in attention, motivation, task proficiency, or perceptual abilities. In sum, these studies provided firm support for the view that older children achieve size constancy (or exhibit overconstancy) for distant objects by using cognitive strategies (on the basis of their knowledge of the effects of distance on image size) that younger children generally do not use.

The perceptual learning hypothesis has also received empirical support. Leibowitz et al. (1967) found that 5- to 10 -year-old children generally exhibited greater underconstancy when viewing distant objects under monocular conditions than under binocular conditions. By contrast, 11-year-old children and adults exhibited size constancy or overconstancy under both monocular and binocular 
viewing conditions when viewing distant objects. Leibowitz et al. concluded from these findings that young children are less sensitive than are adults to the monocular cues - such as linear perspective - that must be detected to achieve accurate size constancy for distant objects.

Although the Leibowitz et al. (1967) results appear to support the perceptual learning hypothesis, it is worth noting that this hypothesis cannot account for some well documented findings. For example, overconstancy is often exhibited by adults (see, e.g., Carlson, 1960; Epstein, 1963; Gilinsky, 1955) and 9- to 10-year-old children (Granrud, 2004) when they estimate the size of a distant object (see also Figure 2). In addition, varying instructions result in widely varying size estimates in adults (see, e.g., Carlson, 1960; Epstein, 1963; Gilinsky, 1955) and older children, but not in younger children (see, e.g., Granrud, 2004; Rapoport, 1967). These findings are consistent with the cognitive supplementation hypothesis but cannot be explained by the perceptual learning hypothesis. It is possible that the cognitive supplementation and perceptual learning hypotheses are both correct. Age-related changes in size constancy performance may result from the development of cognitive strategy use and from increased sensitivity to monocular cues. However, additional research will be needed to determine the importance of cognitive factors versus monocular cue sensitivity in the development of size constancy.

\section{REFERENCES}

BrisLin, R. W., \& Leibowitz, H. W. (1970). The effect of separation between test and comparison objects on size constancy at various agelevels. American Journal of Psychology, 83, 372-376.
CARLson, V. R. (1960). Overestimation in size-constancy judgments. American Journal of Psychology, 73, 199-213.

Epstein, W. (1963). Attitudes of judgment and the size-distance invariance hypothesis. Journal of Experimental Psychology, 66, 78-83.

GiLINSKY, A. S. (1955). The effect of attitude upon the perception of size. American Journal of Psychology, 68, 173-192.

Granrud, C. E. (2004). Visual metacognition and the development of size constancy. In D. T. Levin (Ed.), Thinking and seeing: Visual metacognition in adults and children (pp. 75-95). Cambridge, MA: MIT Press.

Granrud, C. E., \& Morreale, M. A. (2001, April). The role of perceptual knowledge in the development of size constancy. Paper presented at the meeting of the Society for Research in Child Development, Minneapolis, MN.

LeIвоWITZ, H. W. (1974). Multiple mechanisms of size perception and size constancy. Hiroshima Forum for Psychology, 1, 47-53.

Leibowitz, H. W., Pollard, S. W., \& Dickson, D. (1967). Monocular and binocular size-matching as a function of distance at various agelevels. American Journal of Psychology, 80, 263-268.

RAPOPORT, J. L. (1967). Attitude and size judgment in school age children. Child Development, 38, 1187-1192.

RAPOPORT, J. L. (1969). Size-constancy in children measured by a functional size-discrimination task. Journal of Experimental Child Psychology, 7, 366-373.

Rock, I. (1983). The logic of perception. Cambridge, MA: MIT Press.

Shallo, J. (1984). Size perception in children: Evidence for dual mode processing. Unpublished doctoral dissertation, Rutgers University, Newark, NJ.

Shallo, J., \& Rock, I. (1988). Size constancy in children: A new interpretation. Perception, 17, 803-813.

ZeIGLER, H. P., \& LeibowitZ, H. [W.] (1957). Apparent visual size as a function of distance for children and adults. American Journal of Psychology, 70, 106-109.

(Manuscript received May 9, 2005; revision accepted for publication February 13, 2006.) 